\title{
Corrupção: um panorama dos fatores causais em estudos empíricos entre países
}

\author{
Corruption: an overview about causal factors of the empirical studies across countries \\ Corrupción: una visión general de los factores causales en los estudios empíricos entre países
}

Recebido: 07/08/2021 | Revisado: 11/08/2021 | Aceito: 13/08/2021 | Publicado: 16/08/2021

\author{
Nathália Amaral Pereira \\ ORCID: https://orcid.org/0000-0003-1236-8876 \\ Universidade Federal de Viçosa, Brasil \\ E-mail: nathaliaapereira@ufv.br \\ Nálbia de Araújo Santos \\ ORCID: https://orcid.org/0000-0002-6259-1446 \\ Universidade Federal de Viçosa, Brasil \\ E-mail: nalbia@ufv.br \\ Josiel Lopes Valadares \\ ORCID: https://orcid.org/0000-0002-2559-9617 \\ Universidade Federal de Viçosa, Brasil \\ E-mail: josiel.valadares@ufv.br
}

\begin{abstract}
Resumo
Esta pesquisa tem o objetivo de identificar quais as principais evidências encontradas pelas pesquisas empíricas sobre a conceituação, mensuração e as causas da corrupção. Assim, realizou-se uma metanálise qualitativa de estudos empíricos sobre corrupção, sendo consideradas três dimensões: seus fatores causais, sua definição e os índices de mensuração. Como resultado, identificaram-se convergências e peculiaridades acerca do tema na produção científica, indicando uma evolução e amadurecimento na compreensão da concepção sobre corrupção. Nos estudos analisados, os fatores causais da corrupção apontados como significativos são os políticos, sociais, culturais, econômicos e legais de uma sociedade.
\end{abstract}

Palavras-chave: Causas da corrupção; Corrupção; Metanálise.

\begin{abstract}
This research aims to identify the main evidence found by empirical research on the conceptualization, measurement and causes of corruption. Thus, a qualitative meta-analysis of empirical studies on corruption was carried out, considering three dimensions: its causal factors, its definition and measurement indices. As a result, convergences and peculiarities about the theme were identified in scientific production, indicating an evolution and maturation in the understanding of the conception of corruption. In the studies analyzed, the causal factors of corruption identified as significant are the political, social, cultural, economic and legal factors of a society.
\end{abstract}

Keywords: Causes of corruption; Corruption; Meta-analysis.

\section{Resumen}

Esta investigación tiene como objetivo identificar las principales evidencias encontradas por la investigación empírica sobre la conceptualización, medición y causas de la corrupción. Así, se realizó un meta análisis cualitativo de estudios empíricos sobre corrupción, considerando tres dimensiones: sus factores causales, su definición e índices de medición. Como resultado, se identificaron convergencias y peculiaridades sobre el tema en la producción científica, lo que indica una evolución y maduración en la comprensión de la concepción de la corrupción. En los estudios analizados, los factores causales de la corrupción identificados como significativos son los factores políticos, sociales, culturales, económicos y legales de una sociedad.

Palabras clave: Causas de corrupción; Corrupción; Meta análisis.

\section{Introdução}

O fenômeno da corrupção está presente na sociedade, com suas multiformes causas e consequências. O tema tem ganhado cada vez mais espaço nos campos de debates atuais devido a proporção de seus danos à sociedade. A corrupção é reconhecida mundialmente como um problema para gestão pública. Em 2019, o Barômetro Global da Corrupção - América Latina e Caribe, publicado pela Transparência Internacional (TI), constatou que 53\% dos cidadãos consideram que houve um aumento da corrupção em seu país. Ainda, o relatório da organização aponta que a corrupção, principalmente ligada ao setor 
público, coopera para um atraso nos processos de fortalecimento de instituições e princípios importantes à uma democracia efetiva. O estudo da TI destaca que, apesar de haver algum progresso nas ações de combate à corrupção, a grande maioria dos países não alcançou uma maneira eficaz no seu combate.

Esse tema tem atraído a atenção de estudiosos, de diferentes áreas de conhecimento. No entanto, a definição de corrupção assim como sua mensuração são complexas. Desde o trabalho seminal de Becker (1968), um dos primeiros a considerar a corrupção como crime, e também o estudo de Rose-Ackerman (1975), em que abordou sobre os principais aspectos econômicos da corrupção, inúmeras pesquisas se dedicaram a analisar os atos relacionados à corrupção, assim como o comportamento de seus atores envolvidos.

Lambsdorff (2006) ressalta o aumento da elaboração e divulgação de estudos que analisam o fenômeno da corrupção, a relação entre as causas da corrupção e seus aspectos econômicos, políticos ou sociais, além dos mecanismos para seu combate e controle. Becker (1968) abordou a corrupção como um tipo de crime. Pesquisas subsequentes se propuseram a compreender aspectos estruturais e institucionais de países correlacionados a ocorrência de corrupção. Por exemplo, citam-se a origem e a qualidade da estrutura legal (La Porta et al., 1999; Treisman, 2000), os salários do âmbito público (Treisman, 2000; Van Rijckeghem \& Weder, 2001), padrão da estrutura administrativa (Treisman, 2000; Tanzi, 1998) e a estrutura política (Fismam \& Gatti, 2002; Tanzi, 1998), são alguns dos aspectos abordados como relevantes para o entendimento do nível de corrupção de um país. Ademais, a ocorrência de ações que envolvem corrupção tem uma relação intrínseca com as estruturas política, econômica, normativa, social e cultural de uma sociedade.

Palifka e Rose-Ackerman (2016) ressaltam que a cultura e valores fundamentais são diferentes ao redor do mundo, porém há um aspecto que é universal e central quando se busca compreender as experiências desiguais entre países, a motivação humana ou interesse próprio. Assim, cada sociedade tem uma forma específica com a qual define e conduz o interesse próprio, a corrupção endêmica sugere o insucesso ao se canalizar tal aspecto para fins legítimos e produtivos. Para compreender tais falhas no desenvolvimento é necessário compreender de que maneira as instituições lidam com o interesse próprio e como se dá a interação deste, com incentivos atrativos e de natureza pública (Rose-Ackerman \& Palifka, 2016).

A corrupção impacta a legitimidade do governo, influencia a percepção da sociedade em relação ao país que pertence (Avritzer \& Filgueira, 2011). Por conseguinte, para compreender a corrupção é necessário entender o conglomerado de atos, práticas e instituições influentes na sua ocorrência. No entanto, as diversas concepções acerca do fenômeno corrupção, bem como dos atos que a envolvem, indicam a complexidade na sua definição e mensuração. Apesar de existir um grande volume de estudos que envolvem a temática da corrupção, entretanto, trabalhos que se dedicam exclusivamente a um levantamento teórico ou revisão de literatura tem menor representatividade. Portanto destaca-se a relevância da elaboração de estudos dessa natureza para auxiliar pesquisas futuras na área.

Nesse contexto, com o intuito de preencher a lacuna presente na literatura buscou-se desvendar os sentidos e direções de estudos sobre corrupção, assim este trabalho tem como questão principal: quais as principais contribuições de estudos

empíricos publicados entre 2005 e 2020 para ampliar a compreensão sobre os fatores causais da corrupção e para conceituação e mensuração desse fenômeno?

A presente pesquisa tem a pretensão de contribuir para discussão e compreensão dos fatores causais da corrupção, sua conceituação e mensuração, bem como para um melhor direcionamento das estratégias de combate da corrupção, do desenvolvimento dos mecanismos de controle e também para a formulação de políticas públicas. A análise realizada é relevante e oportuna para a percepção de questões importantes para o aperfeiçoamento de investigações futuras. 


\section{Metodologia}

Conforme objetivo estabelecido aplicou-se a técnica de meta-análise para realizar uma revisão sistemática da literatura da produção científica sobre o tema corrupção e seus fatores causais, especificamente no âmbito público. A técnica foi realizada por ser importante para se obter uma melhor compreensão sobre o atual desenvolvimento dos conhecimentos sobre um determinado tema (Bento, 2012).

O procedimento utilizado nessa pesquisa foi o levantamento de artigos que versam sobre a corrupção, especificamente sobre os fatores causais desse fenômeno. Com intuito de identificar os estudos relevantes para inclusão na análise, realizou-se uma busca por artigos nas bases: Scientific Periodicals Electronic Library - SPELL e Web Science em que foi analisada a Principal Coleção do Web of Science. Foram selecionados para a busca os seguintes descritores: "causas”, "corrupção”; para a busca na base da SPELL; e "causes", "corruption" na base da Web of Science, juntamente com os termos boleanos OR ou AND.

O corte temporal estabelecido foi de janeiro de 2005 a junho de 2020 e realizada a busca nas bases de dados definidas anteriormente. Essa etapa inicial de busca resultou em 145 artigos no Scientific Periodicals Electronic Library - SPELL e 473 no Web of Science, totalizando em 618 artigos. O levantamento de dados foi realizado entre os meses de junho e agosto de 2020.

Após a coleta de dados foram estabelecidos os critérios com o objetivo de delimitar o viés da escolha dos artigos, condensar e avaliar criticamente os estudos importantes para coletar e analisar os seus dados (Galvão, Sawada, Trevisan, 2004). Assim, realizou-se leituras criteriosas dos títulos, resumos e palavras-chaves e, quando necessário, o artigo foi lido por completo para analisar sua adequação ao escopo da pesquisa. Nessa etapa, foram descartados 547 artigos em virtude dos estudos, apesar do assunto central ser corrupção, não abordarem os fatores causais do fenômeno. Como requisitos para seleção dos artigos para análise considerou-se que as pesquisas analisavam pelo menos um antecedente ou causa da corrupção, são estudos empíricos e realizaram análises entre países. Esses critérios possibilitaram análises comparativas mais robusta dos resultados, já que esses estudos entre países envolvem contextos com maiores dissimilares, permitindo a percepção do delineamento do fenômeno corrupção em diversos cenários. Deste modo, foram descartadas 36 revisões de literatura e ensaios teóricos, 15 estudos que não abordavam mais de um país. Após essa etapa, foram selecionados os 20 artigos que compuseram a amostra final e, portanto, constituindo a base dessa pesquisa,

Os artigos componentes da base dessa pesquisa são apresentados no Quadro 1, a seguir. 
Quadro 1 - Artigos selecionados para análise.

\begin{tabular}{|c|c|c|c|}
\hline & Ano & $\begin{array}{c}\text { Título } \\
\end{array}$ & Local de Publicação \\
\hline $\mathbf{A 1}$ & 2005 & $\begin{array}{l}\text { Electoral rules and constitutional structures as constraints on } \\
\text { corruption. }\end{array}$ & British journal of political science \\
\hline A2 & 2005 & Accountability and corruption: Political institutions matter. & Economics \& politics \\
\hline $\mathbf{A 3}$ & 2006 & Empirical determinants of corruption: A sensitivity analysis. & Public Choice \\
\hline A4 & 2007 & $\begin{array}{l}\text { Causes of corruption: a survey of cross-country analyses and extended } \\
\text { results. }\end{array}$ & Springer \\
\hline A5 & 2009 & Instability and the Incentives for Corruption. & Economics \& Politics \\
\hline A6 & 2009 & $\begin{array}{l}\text { Do existing corruption levels matter in controlling corruption? Cross- } \\
\text { country quantile regression estimates. }\end{array}$ & Journal of Development Economics \\
\hline A7 & 2010 & Causes of corruption: History, geography and government. & Journal of Policy Modeling \\
\hline $\mathbf{A 8}$ & 2012 & $\begin{array}{l}\text { Government size, democracy, and corruption: An empirical } \\
\text { investigation. }\end{array}$ & Economic Modelling \\
\hline A9 & 2013 & Religion, corruption, and the rule of law. & Journal of Money, Credit and Banking \\
\hline $\mathbf{A 1 0}$ & 2014 & $\begin{array}{l}\text { Government size, nonprofit sector strength, and corruption: a cross- } \\
\text { national examination. }\end{array}$ & The American Review of Public Administration \\
\hline A11 & 2015 & $\begin{array}{l}\text { Corruption, political instability and economic development in the } \\
\text { Economic Community of West African states (ECOWAS): Is there a } \\
\text { causal relationship. }\end{array}$ & Contemporary Economics \\
\hline $\mathbf{A 1 2}$ & 2015 & $\begin{array}{l}\text { Keeping both corruption and the shadow economy in check: the role } \\
\text { of decentralization. }\end{array}$ & International Tax and Public Finance, \\
\hline $\mathbf{A 1 3}$ & 2017 & Corruption in Transition Economies: Cause or Effect? & $\begin{array}{l}\text { Zagreb International Review of Economics and } \\
\text { Business }\end{array}$ \\
\hline $\mathbf{A 1 4}$ & 2018 & $\begin{array}{l}\text { Relações entre Corrupção Percebida e Transparência Orçamentária: } \\
\text { um estudo com abordagem cross-national. }\end{array}$ & Revista Contemporânea de Contabilidade \\
\hline $\mathbf{A 1 5}$ & 2018 & $\begin{array}{l}\text { Corruption causes inequality, or is it the other way around? An } \\
\text { empirical investigation for a panel of countries. }\end{array}$ & Economic Analysis and Policy \\
\hline A16 & 2018 & $\begin{array}{l}\text { Mais capacidade estatal, menos corrupção? Uma análise para a } \\
\text { América Latina (1996-2015) }\end{array}$ & Revista de Sociologia e Política \\
\hline A17 & 2019 & $\begin{array}{l}\text { Corrupção governamental e difusão do acesso à Internet: evidências } \\
\text { globais }\end{array}$ & Revista de Administração Pública \\
\hline A18 & 2019 & $\begin{array}{l}\text { The effects of social media use on control of corruption and } \\
\text { moderating role of cultural tightness-looseness }\end{array}$ & Government Information Quarterly \\
\hline A19 & 2019 & $\begin{array}{l}\text { Causality between income inequality and corruption in OECD } \\
\text { countries. }\end{array}$ & World Development Perspectives \\
\hline $\mathbf{A 2 0}$ & 2020 & $\begin{array}{l}\text { Efeito conjunto da democracia e da liberdade econômica sobre a } \\
\text { corrupção. }\end{array}$ & Revista de Administração Pública \\
\hline
\end{tabular}

Fonte: Dados da pesquisa.

O Quadro 1 apresenta em sua primeira coluna uma legenda com intuito de auxiliar na identificação dos artigos analisados na pesquisa citados nas seções e quadros subsequentes. A seguir, à direita está representado respectivamente: o ano de publicação, o título do artigo e o periódico de publicação.

Para análise dos dados optou-se pela aplicação da técnica de análise de conteúdo, possibilitando dessa forma a construção dos resultados de forma qualitativa. Para tanto, e em conformidade com Bardin (2004) as fases da análise de conteúdo organizaram-se em: análise prévia (pré-seleção do material por meio de busca e leitura superficial dos documentos); exploração do material (seleção final do material através da análise e leitura completa dos mesmos); e tratamento dos resultados (aplicação de uma análise qualitativa do material elegido na fase antecedente). A definição de categorias são procedimentos fundamentais para a análise de conteúdo, portanto nessa pesquisa optou-se pela grade de categorias abertas, pela sua flexibilidade, em que as categorias de análise são definidas durante o desenvolvimento da pesquisa (Laville \& Dionne, 1999; Vergara, 2006). 


\section{Resultados e Discussão}

\subsection{Tratamento dos fatores causais da corrupção na produção científica}

Nas últimas décadas foram elaboradas pesquisas sobre o tema corrupção por diversos pesquisadores de variados campos do conhecimento e de diferentes perspectivas teóricas. As pesquisas acerca dos fatores causais da corrupção possuem um delineamento complexo como destaca Lambsdorffl (2006), que atribui tal complexidade ao fato de muitas causas também se mostrarem como consequências. Tendo em conta as observações acima, na sequência desse tópico procurou-se identificar as diferentes dimensões utilizadas na análise dos fatores causais, a fim de compreender de que forma a corrupção e suas causas são enquadradas teoricamente.

A perspectiva econômica tem predominado nos estudos acerca dos fatores causais de corrupção nos últimos anos. Nessa perspectiva os estudos concentram-se na maneira com que as instituições econômicas exercem influência no nível de corrupção de um país (Judge et al.2011). Há pesquisadores que dedicaram seus esforços aos estudos da relação entre a corrupção e o desenvolvimento econômico ou prosperidade econômica (Serra, 2006; Pellegrini \& Gerlagh, 2007; Billger \& Goel, 2009; Goel \& Nelson, 2010; Abu et al., 2015; Arapović et al., 2017; Policardo \& Carrera, 2018; Viana et al., 2020).

Ao buscar verificar a robustez de evidências empíricas anteriormente encontradas na literatura Serra (2006), identifica que as sociedades mais ricas têm menor propensão de serem corruptas, se comparada com as mais pobres. Os autores Pellegrini e Gerlagh (2007) ao testar hipóteses previamente estabelecidas por pesquisas empíricas, também encontraram evidências que apoiam o pressuposto de que níveis de renda mais altos, impactam em um nível mais baixo de corrupção. Tais resultados são corroborados ainda por Billger e Goel (2009), em sua investigação sobre os determinantes da corrupção e sua distribuição condicional entre as nações e por Goel e Nelson (2010) que examinaram o papel dos fatores históricos, das influências geográficas e do governo sobre a corrupção. Viana et al. (2020) que analisou o efeito conjunto da democracia e da liberdade econômica sobre a corrupção, também encontra evidências que em países mais pobres existe um nível mais elevado de corrupção.

Os autores Abu et al. (2015) examinaram a relação causal existente entre a corrupção, a instabilidade política e o desenvolvimento econômico, em relação a esse último identificaram que em países com um fraco desempenho econômico há um maior grau de corrupção. Arapović et al. (2017) por sua vez, buscaram investigar a relação entre corrupção, o crescimento econômico e os gastos do governo em 14 economias em transição. Como resultado identificou-se um elo causal bilateral entre o desenvolvimento econômico e a corrupção. No entanto, os autores concluem o estudo com ressalvas a respeito dessa causalidade, que serão abordadas mais à frente nessa pesquisa.

A desigualdade de renda é outro fator econômico tratado na literatura (Policardo \& Carrera, 2018; Policardo et al., 2019). Ao investigar o efeito desse fator sobre a corrupção, e averiguar a relação causal entre os mesmos, os autores Policardo e Carrera (2018) identificaram que a desigualdade de renda aumenta consistentemente a corrupção. Posto que, pessoas com menos riquezas estariam mais propensas a se envolver em atos de corrupção na busca por renda. Enquanto os ricos teriam uma maior motivação e oportunidade de praticarem corrupção, devido maior vulnerabilidade dos pobres e, consequentemente, uma menor capacidade de monitoramento e responsabilização (Policardo \& Carrera, 2018). Já Policardo et al. (2019) acrescentam que quando a população julga a desigualdade de renda como injusta, a corrupção pode tornar-se uma resposta a essa distribuição "injusta" existente. Tal preposição e resultado da análise do efeito da corrupção sobre a desigualdade de renda e sua relação nos países da OCDE, realizada pelos autores.

A literatura que compõe a base dessa pesquisa aponta a liberdade econômica como um aspecto que influi significativamente na corrupção (Serra, 2006; Billger \& Goel, 2009; Viana et al., 2020). No entanto, existem divergências em relação aos resultados encontrados, que serão retomados mais adiante. Outros fatores econômicos são considerados como 
determinantes da corrupção, porém, serão abordados nessa pesquisa apenas os achados na produção acadêmica selecionada para base de dados analisada.

A perspectiva ou dimensão política e legal da corrupção também é retratada por estudos analisados nessa pesquisa (Kunicová \& Rose-Ackerman, 2005; Lederman et al., 2005; Pellegrini \& Gerlagh, 2007; Goel \& Nelson, 2010; Judge et al., 2011; Abu et al., 2015; Dell'anno \& Teobaldelli, 2015; Reis et al., 2019; Campante et al., 2019), estes buscam explicar a corrupção sob o foco regulamentar/legal e governamental, nesse sentido se baseiam na influência exercida por instituições políticas e legais no nível de corrupção de um país (Judge et al., 2011). A estrutura política e de governo de uma país é indicada como um fator causal da corrupção (Kunicová \& Rose-Ackerman, 2005; Pellegrini \& Gerlagh, 2007). As autoras Kunicová e Rose-Ackerman (2005) investigaram de que forma os diferentes sistemas eleitorais e suas regras restringem a corrupção em um país. Encontraram evidencias empíricas que indicam que sistemas de repúblicas, especialmente associadas ao presidencialismo por representação proporcional, geralmente estão acompanhados de elevados níveis de corrupção.

Outro fator causal da corrupção é a descentralização do governo (Kunicová \& Rose-Ackerman, 2005; Lederman et al., 2005; Goel \& Nelson, 2010; Dell'anno \& Teobaldelli, 2015; Reis et al., 2019), no entanto, os achados na literatura são conflitantes. Os autores Lederman et al. (2005) em sua pesquisa examinaram determinantes da corrupção, com foco principal nas instituições políticas. Encontrou-se evidencias de que os tipos de descentralização exercem diferentes influências sobre a corrupção. Quando ocorre a descentralização relacionada a concessão de uma maior autonomia aos estados, em especial de se legislar, os níveis de corrupção se mostram maiores. Já quando a descentralização ocorre relacionada a gastos entre os níveis do governo, há uma tendência de redução da corrupção, conforme resultados encontrados por Lederman et al. (2005). Tal preposição também é apoiada por Reis et al. (2019) ao verificar se a transparência orçamentária, os fatores sociais, econômicos, e políticos institucionais podem explicar o nível percebido de corrupção. Ainda nesse sentido Kunicová e Rose-Ackerman (2005) demonstram que o federalismo é, geralmente, acompanhado de um alto nível de corrupção. Os autores Dell’Anno e Teobaldelli (2015) em um trabalho posterior, em que avaliaram os efeitos da descentralização governamental na economia informal e na corrupção, encontram evidências empíricas a respeito do efeito redutor da descentralização sobre a corrupção.

O tamanho do governo também se revela um fator influente no nível de corrupção (Billger \& Goel, 2009; Goel \& Nelson, 2010; Kotera et al., 2012). Os estudiosos Goel e Nelson (2010) demonstram em sua pesquisa que governos maiores resultaram em um maior nível de corrupção. Em um trabalho posterior Kotera et al. (2012) verificaram a relação entre a corrupção e o tamanho do governo. Encontraram evidencias empíricas que apontam que um governo maior eleva a corrupção se há também níveis baixos de democracia. Caso contrário, o efeito é reverso. Nesse sentido, verificamos que os achados na produção acadêmica sobre o tamanho do governo como um fator causal da corrupção possuí resultados imprecisos.

A eficiência das instituições políticas assim como questões de instabilidade política é apontada como fatores causais da corrupção (Lederman et al., 2005; Serra, 2006; Abu et al., 2015; Campante et al., 2019). Os autores Abu et al. (2015) atribuem os altos índices de corrupção nos países da CEDEA, ao longo período de instabilidade política nos países. Os pesquisadores Lederman et al. (2005) e Serra (2006) confirmam a relação causal entre a instabilidade política e corrupção. Em países caracterizados pela maior instabilidade política a tendência é de se tornarem mais corruptos. Campante et al. (2009) ao examinar de que maneira a estabilidade política influi nos incentivos para envolvimento em comportamento corrupto, os autores encontraram evidências de que onde há níveis mais elevados de instabilidade o agente político está mais disposto a se envolver em atos corruptos. Os autores adotam por estabilidade política, a estabilidade do cargo que os titulares políticos são expostos.

A intervenção estatal na economia é indicada também como um fator causal da corrupção. Uma sociedade ou região onde há muita regulamentação tem maior probabilidade de que ocorra práticas corruptas, se associadas a uma burocracia e estrutura política ineficiente, bem como a origem do sistema constitucional ou de lei (Goel \& Nelson, 2010; Nascimento, 2018). O pesquisador Nascimento (2018), encontra evidências empíricas de que a eficácia política impacta negativamente os níveis de 
corrupção, em sua investigação acerca das relações entre a difusão da internet, voz e accountability, dimensão cultural, corrupção e eficácia governamental.

A perspectiva ou dimensão social e cultural é apresentada por alguns autores (Lederman et al., 2005; Serra, 2006; Pellegrini \& Gerlagh, 2007; North et al., 2013, Nascimento, 2018; Nascimento et al., 2018; Tang et al., 2019). Pesquisas com esse foco desempenho um papel importante, ao destacar as formas heterogêneas como a corrupção se manifesta entre as sociedades. Os aspectos culturais podem contribuir para elucidar, em parte, a grande variação no nível de corrupção entre os países e regiões. A forma com que uma sociedade lida com a corrupção e seus agentes, e o nível de tolerância com essa situação está relacionada com a cultura de uma sociedade, suas crenças, religião predominante e comportamento social (Pellegrini \& Gerlagh, 2007; North et al., 2013, Nascimento, 2018; Nascimento et al., 2018; Tang et al., 2019). A participação civil, a democracia e a forma como a imprensa lida com o fenômeno da corrupção também são apresentados por estudos como fatores de relacionamento causal com a corrupção (Lederman et al., 2005). A herança colonial também aparece fortemente relacionada ao nível de corrupção (Serra, 2006)

No Quadro 2 estão apresentados os artigos que constituem a base de dados, com informações da esquerda para direita: legenda de identificação (segundo designação no Quadro 1), objetivo do estudo, método aplicado e os principais resultados.

Quadro 2 - Tratamento dos fatores causais da corrupção na produção científica.

\begin{tabular}{|c|c|c|c|}
\hline & Objetivo & Método & Principais Resultados \\
\hline A1 & $\begin{array}{l}\text { Investigar como os diferentes } \\
\text { sistemas eleitorais restringem a } \\
\text { busca por renda corrupta, } \\
\text { mantendo constante outros fatores } \\
\text { políticos, econômicos e sociais. }\end{array}$ & $\begin{array}{l}\text { Foram utilizados dados de } 94 \text { países que } \\
\text { caracterizam os países em termos de nível de } \\
\text { corrupção e foram acrescentadas informações } \\
\text { outras varáveis políticas e econômicas. Aplicou-se } \\
\text { um modelo econométrico de regressão. }\end{array}$ & $\begin{array}{l}\text { As regras eleitorais influenciam a } \\
\text { interação e a dinâmica entre os eleitores } \\
\mathrm{e} \text { atores políticos. Os sistemas de } \\
\text { república, em especial quando em } \\
\text { conjunto ao presidencialismo possuem } \\
\text { níveis elevados de corrupção. }\end{array}$ \\
\hline A2 & $\begin{array}{l}\text { Compreender os determinantes } \\
\text { fundamentais da corrupção, } \\
\text { concentrando-se nas instituições } \\
\text { políticas que determinam as } \\
\text { políticas específicas, bem como os } \\
\text { resultados políticos. }\end{array}$ & $\begin{array}{l}\text { Utilizando um painel de vários países foi realizada } \\
\text { uma análise multivariada dos dados, verificando a } \\
\text { robustez dos resultados pela análise transversal. }\end{array}$ & $\begin{array}{l}\text { Há uma redução sistemática da } \\
\text { corrupção relacionado a democracia, } \\
\text { liberdade de imprensa e sistemas } \\
\text { parlamentares. A descentralização } \\
\text { relacionada aos gastos governamentais, } \\
\text { tendem a aumentar a corrupção. }\end{array}$ \\
\hline $\mathbf{A 3}$ & $\begin{array}{l}\text { Analisar um conjunto de } \\
\text { evidências empíricas anteriores a } \\
\text { fim de verificar a robustez dos } \\
\text { dados apresentados na literatura. }\end{array}$ & $\begin{array}{l}\text { Foi utilizado dados de } 62 \text { países, desenvolvidos e } \\
\text { em desenvolvimento. Foram testadas } 16 \text { variáveis } \\
\text { quanto a robustez pela Análise de Limites } \\
\text { Extremos (EBA). }\end{array}$ & $\begin{array}{l}\text { Países ricos tendem a ter menor } \\
\text { corrupção, que os mais pobres. Uma } \\
\text { maior instabilidade política aumenta a } \\
\text { corrupção, bem como em países com } \\
\text { forte herança colonial. }\end{array}$ \\
\hline $\mathbf{A 4}$ & $\begin{array}{l}\text { Levantar e avaliar a literatura } \\
\text { empírica sobre as fontes de } \\
\text { corrupção, e calcular a partir } \\
\text { dessas uma estimativa própria das } \\
\text { fontes de corrupção. }\end{array}$ & $\begin{array}{l}\text { Foi utilizado dados do Banco Mundial na análise } \\
\text { central e o Índice da Transparency International } \\
\text { para verificação da robustez. Por meio de uma } \\
\text { regressão dos mínimos quadrados fornecem uma } \\
\text { estimativa de corrupção entre os países. }\end{array}$ & $\begin{array}{l}\text { A democracia tem um efeito redutor da } \\
\text { corrupção, enquanto a instabilidade } \\
\text { política tende a aumentar. A liberdade de } \\
\text { imprensa e difusão de jornais e revistas } \\
\text { baixam o nível de corrupção. }\end{array}$ \\
\hline$\overline{A 5}$ & $\begin{array}{l}\text { Examinar de que maneira a } \\
\text { estabilidade política influi nos } \\
\text { incentivos para envolvimento em } \\
\text { comportamento corrupto. }\end{array}$ & $\begin{array}{l}\text { Os autores propõem um modelo que gera uma } \\
\text { relação estilizada em forma de U entre a corrupção } \\
\text { e estabilidade política, com base na interação entre } \\
\text { o efeito horizonte e o efeito demanda. }\end{array}$ & $\begin{array}{l}\text { Há um efeito horizonte entre a } \\
\text { estabilidade de posição do agente, } \\
\text { menos estabilidade mais corrupção. } \\
\text { Onde há maior estabilidade a relação } \\
\text { apresenta-se em padrão de U. }\end{array}$ \\
\hline A6 & $\begin{array}{l}\text { Analisar os determinantes de } \\
\text { corrupção a fim de compreender se } \\
\text { o nível de corrupção impacta na } \\
\text { forma como estes se manifesta. }\end{array}$ & $\begin{array}{l}\text { Usando dados transversais de países aplicando a } \\
\text { técnica de regressão quartis e método dos mínimos } \\
\text { quadrados ordinários (OLS) para análise dos } \\
\text { dados. }\end{array}$ & $\begin{array}{l}\text { Uma maior prosperidade econômica } \\
\text { reduz a corrupção, à medida que o nível } \\
\text { democrático se torna maior a corrupção } \\
\text { reduz. }\end{array}$ \\
\hline A7 & $\begin{array}{l}\text { Examinar o papel dos fatores } \\
\text { históricos, } \\
\text { geográficas e do governo sobre a } \\
\text { corrupção. }\end{array}$ & $\begin{array}{l}\text { O conjunto de amostras de dados combinados } \\
\text { inclui cerca de } 100 \text { países e } 3 \text { períodos de tempos: } \\
\text { 1995-1997, 1998-2000, e 2001-2003. Foi aplicado } \\
\text { uma regressão linear com } 3 \text { variáveis de controle } \\
\text { incorporadas em estudos empíricos anteriores. }\end{array}$ & $\begin{array}{l}\text { A distribuição populacional impacta } \\
\text { sobre a corrupção, as influências } \\
\text { históricas e geográficas são exógenas e } \\
\text { uma maior intervenção do Estado na } \\
\text { economia eleva a corrupção. }\end{array}$ \\
\hline A8 & $\begin{array}{l}\text { Examinar a relação entre a } \\
\text { corrupção e o tamanho do governo } \\
\text { usando dados de } 82 \text { países para o } \\
\text { período de } 1995 \text { a } 2008 \text {. }\end{array}$ & $\begin{array}{l}\text { Foi aplicada como técnica de análise de dados o } \\
\text { método dos mínimos quadrados ordinários (OLS) } \\
\text { com uma verificação de análise de robustez com } \\
\text { método generalizado dos momentos (GMM). }\end{array}$ & $\begin{array}{l}\text { Em países que possui um nível baixo de } \\
\text { democracia um governo maior aumenta } \\
\text { a corrupção. }\end{array}$ \\
\hline
\end{tabular}




\begin{tabular}{|c|c|c|c|}
\hline A9 & $\begin{array}{l}\text { Examinar se a cultura religiosa } \\
\text { dominante de uma nação afeta o } \\
\text { Estado de direito e o grau de } \\
\text { controle sobre a corrupção. }\end{array}$ & $\begin{array}{l}\text { Os autores estimaram várias regressões lineares do } \\
\text { índice de Estado de Direito e Índice de Controle de } \\
\text { Corrupção em um conjunto de variáveis, em } \\
\text { seguida realizaram teste de hipóteses em pares } \\
\text { sobre as estimativas de coeficientes para cada } \\
\text { variável de religião. }\end{array}$ & $\begin{array}{l}\text { O cristianismo ortodoxo em um país em } \\
1900 \text { está associado a níveis mais altos } \\
\text { de corrupção hoje, enquanto o } \\
\text { protestantismo está associado a níveis } \\
\text { mais baixos. }\end{array}$ \\
\hline A10 & $\begin{array}{l}\text { Avaliar a validade empírica, por } \\
\text { meio da análise transnacional, do } \\
\text { argumento popular "grande } \\
\text { governo é mau governo" com a } \\
\text { relação corrupta. }\end{array}$ & $\begin{array}{l}\text { Foi aplicado na análise dos dados o método de } \\
\text { regressão dos mínimos quadrados ponderados } \\
\text { (WLS) e para separa as relações de causa-efeito } \\
\text { das variáveis se aplicou a regressão ponderada de } \\
\text { mínimos quadrados em } 2 \text { estágios (2SLS). }\end{array}$ & $\begin{array}{l}\text { O tamanho do governo pode reduzir o } \\
\text { nível de corrupção. Um governo maior } \\
\text { resulta em níveis menores de corrupção. }\end{array}$ \\
\hline A11 & $\begin{array}{l}\text { Examinar a relação causal entre } \\
\text { corrupção, instabilidade política e } \\
\text { desenvolvimento econômico na } \\
\text { Comunidade Económica dos } \\
\text { Estados da África Ocidental } \\
\text { (CEDEAO). }\end{array}$ & $\begin{array}{l}\text { Aplicou-se o teste de causalidade de Granger } \\
\text { dentro de uma estrutura multivariada de integração } \\
\text { e correção de erros no período de 1996-2012 pra } \\
\text { países da CEDEAO. }\end{array}$ & $\begin{array}{l}\text { Foi encontrada uma causalidade positiva } \\
\text { partindo da instabilidade política e } \\
\text { desenvolvimento econômico para } \\
\text { corrupção a longo prazo. }\end{array}$ \\
\hline A12 & 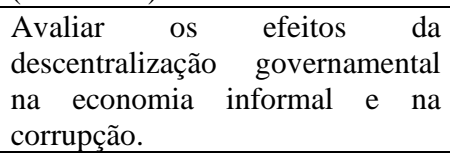 & $\begin{array}{l}\text { A análise baseia-se em uma base de dados de } 145 \\
\text { países que inclui diferentes índices de } \\
\text { descentralização, corrupção e economia informal. } \\
\text { Foram como técnica de análise a regressão linear. }\end{array}$ & $\begin{array}{l}\text { Em países como maior descentralização } \\
\text { a corrupção e a economia informal } \\
\text { tendem a ser menores. }\end{array}$ \\
\hline A13 & $\begin{array}{l}\text { Investigar a relação intertemporal } \\
\text { entre corrupção, crescimento } \\
\text { econômico e gastos públicos para } \\
14 \text { economias em transição } \\
\text { durante o período } 1995-2013 \text {. }\end{array}$ & $\begin{array}{l}\text { Foi aplicado a base de dados o teste de causalidade } \\
\text { de Granger, com intuito de verificar a relação } \\
\text { causal entre a variáveis. }\end{array}$ & $\begin{array}{l}\text { Apresentou ela causal bilateral de } \\
\text { Granger entre corrupção } \\
\text { desenvolvimento econômico. }\end{array}$ \\
\hline A14 & $\begin{array}{l}\text { Verificar se a transparência } \\
\text { orçamentária, fatores sociais, } \\
\text { econômicos, e políticos } \\
\text { institucionais podem explicar o } \\
\text { nível percebido de corrupção nos } \\
\text { países analisados. }\end{array}$ & $\begin{array}{l}\text { A base de dados é composta por } 82 \text { países com } \\
\text { dados de corte transversal referente ao ano de } \\
\text { 2010. Para análise é aplicada a técnica estatística } \\
\text { dos modelos de regressão para dados de contagem: } \\
\text { Poisson ou binominal negativo. }\end{array}$ & $\begin{array}{l}\text { Os fatores analisados que que } \\
\text { demonstraram poder de explicação da } \\
\text { corrupção estão a transparência } \\
\text { orçamentária, as variáveis sociais, } \\
\text { econômicas e político-institucionais. }\end{array}$ \\
\hline A15 & $\begin{array}{l}\text { Investigar o efeito da desigualdade } \\
\text { de renda sobre a corrupção e sua } \\
\text { relação causal. }\end{array}$ & $\begin{array}{l}\text { Com base no conjunto de dados de } 50 \text { países de } \\
1995 \text { a 2015, aplicaram o modelo dinâmico de } \\
\text { dados em painel método generalizado dos } \\
\text { momentos (GMM). }\end{array}$ & $\begin{array}{l}\text { Quando há uma desigualdade de renda } \\
\text { alta a corrupção aumenta } \\
\text { significativamente. }\end{array}$ \\
\hline A16 & $\begin{array}{l}\text { Testar a hipótese de que a } \\
\text { capacidade estatal diminuí os } \\
\text { níveis de corrupção na América } \\
\text { Latina no período entre os anos de } \\
1996 \text { e 2015. }\end{array}$ & $\begin{array}{l}\text { A técnica de análise de dados aplicada as hipóteses } \\
\text { elaboradas foram estimadas painéis de dados e o } \\
\text { Modelo econométrico do tipo linear de dados de } \\
\text { painel com erros padrão corrigidos (PCSE). }\end{array}$ & $\begin{array}{l}\text { Uma maior capacidade estatal aumenta } \\
\text { os controles de corrupção, influenciando } \\
\text { na redução da mesma }\end{array}$ \\
\hline A17 & 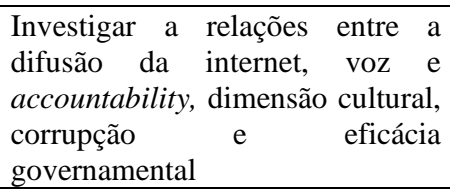 & $\begin{array}{l}\text { Compõem a base dados } 117 \text { nações no período de } \\
2000 \text { a } 2014 \text {. Adotaram a técnica de Análise } \\
\text { Robusta de caminho para análise de dados. }\end{array}$ & $\begin{array}{l}\text { Uma maior difusão da internet resulta } \\
\text { em níveis menores de corrupção } \\
\text { governamental }\end{array}$ \\
\hline A18 & $\begin{array}{l}\text { Determinar se o nível de uso de } \\
\text { mídia social de um país impacta no } \\
\text { controle da corrupção e na eficácia } \\
\text { governamental }\end{array}$ & $\begin{array}{l}\text { Foram analisados um conjunto de dados em painel } \\
\text { de } 5 \text { anos (2001-2015) de } 62 \text { países }\end{array}$ & $\begin{array}{l}\text { Um maior uso das mídias sociais resulta } \\
\text { em um menor nível de corrupção }\end{array}$ \\
\hline A19 & $\begin{array}{l}\text { Investigar o efeito da corrupção } \\
\text { sobre a desigualdade de renda e } \\
\text { sua relação causal nos países da } \\
\text { Organização para a Cooperação e } \\
\text { Desenvolvimento Econômico } \\
\text { (OCDE). }\end{array}$ & $\begin{array}{l}\text { São analisados } 34 \text { países da OCDE entre o período } \\
\text { de } 1995-2011 \text { e aplicada uma regressão estimada. }\end{array}$ & $\begin{array}{l}\text { Os resultados revelam que a } \\
\text { desigualdade de renda aumenta a } \\
\text { corrupção, ao mesmo que a corrupção } \\
\text { afeta a desigualdade }\end{array}$ \\
\hline A20 & $\begin{array}{l}\text { Examinar o efeito conjunto da } \\
\text { democracia e da liberdade } \\
\text { econômica sobre a corrupção. }\end{array}$ & $\begin{array}{l}\text { Utilizando as bases de dados de } 160 \text { países nos } \\
\text { anos de } 2010-2016 \text {, considerando diversas fontes } \\
\text { de informação para o conjunto de variáveis } \\
\text { econômicas, culturais, históricas e institucionais. } \\
\text { Foi utilizado um modelo de regressão. }\end{array}$ & $\begin{array}{l}\text { Quanto um nível maior de democracia e } \\
\text { liberdade econômica se mostram } \\
\text { relevantes na redução da corrupção. }\end{array}$ \\
\hline
\end{tabular}

Fonte: Dados da pesquisa. 


\subsection{Definições e mensuração da corrupção}

Diferentes definições de corrupção surgem, algumas fundamentadas na moral e outras com bases mais legais e econômicas. O fenômeno da corrupção pode se manifestar em diferentes comportamentos: o nepotismo, as fraudes, o suborno, os desvios de verbas e entre outros atos, estes constituem a diversidade de ações que envolvem o conceito de corrupção (Brei, 1996b; Rose-Ackerman \& Palifka, 2016). Porém, existem divergências quanto a conceituação do fenômeno entre os autores que possuem trabalhos sobre o tema, um dos pontos críticos de tal situação diz respeito a dificuldade de conceituação precisa do ato de corrupção (Brei, 1996b).

As autoras Kunicová e Rose Ackerman (2005) adotam a concepção de corrupção como "the misuse of public office for private financial gain by an elected official" (Kunicová \& Rose-Ackerman, 2005, p.577). Ressaltam a questão do enriquecimento de forma ilícita e oculta, como ponto chave de tal definição e conferem destaque as raízes institucionais do fenômeno e sua natureza moral e jurídica. Ainda nesse sentido, há estudos que definem a corrupção como um exercício, uso ou abuso de um poder ou cargo público, para ganhos privados (Goel \& Nelson, 2010; North et al., 2013) onde há por parte dos agentes (tanto corruptor quanto o corrupto) desrespeito pelas regras que regem suas ações (North et al., 2013). Esses agentes exploram a posição de intermediários e obtém vantagens do poder confiado, agindo em interesse próprio (Nascimento et al., 2019).

Autores como Pellegrini e Gerlagh (2011), Dell'Anno e Teobladelli (2013), Themudo (2014) e Tang et al (2019) utilizam a concepção de corrupção como "abuso de um poder confiado para ganho privado". Essa definição propagada pela Transparência Internacional é frequentemente utilizada em pesquisas. Além disso, Themudo (2014) e Tang et al. (2019) referemse à corrupção como uma espécie de reflexo de diversos comportamentos, como suborno, peculato, extorsão, nepotismo, tomada de decisões tendenciosas de agentes e falta de transparência. Dell'Anno e Teobaldelli (2013) tratam a questão do ganho privado como um resultado da extração de verba pelos agentes corruptos. Já os autores Campante et al. (2009) abordam a corrupção de duas maneiras em seu trabalho. Uma delas se refere a apropriação indevida de propriedades do governo para enriquecimento próprio e a segunda define a corrupção como suborno.

$\mathrm{Na}$ literatura científica ainda encontramos estudos que utilizam a concepção de corrupção em sua natureza criminosa, como é o caso dos estudos desenvolvidos por Lederman et al. (2005) e Billger e Goel (2009), Goel e Nelson (2010). Apesar do tratamento como crime adotado nos estudos, a prática da corrupção diferencia-se dos outros crimes no sentido que se configura um fenômeno político (Lederman et al., 2005). Nele os agentes, como indivíduos racionais, calculam os resultados de fazer parte de um ato corrupto ou não (Billger \& Goel, 2009, Goel \& Nelson, 2010). Nesse sentido Reis et al. (2019) apresentam as variadas concepções sobre corrupção e adotam a corrupção como um problema público que envolve a ação de agentes públicos.

O debate acerca da definição de corrupção é assunto de discussões em conferências e reuniões há tempos (Tanzi, 1998). O fenômeno tem sido abordado como uma questão social que se tornou pauta não apenas a nível local, mas internacionalmente. Apesar de não haver uma definição universal quando se trata de corrupção, o que prevalece é que tal situação gera efeitos negativos à sociedade (Tang et al., 2019).

Dada a importância e abrangência da corrupção, sua mensuração é fundamental. Na literatura, diversas formas de mensurar a corrupção podem ser encontradas, bem como inúmeras perspectivas de análise e índices. Serão abordados, os índices utilizados pelos trabalhos que compõe a base desses estudos.

Um dos principais indicadores utilizados nas pesquisas empíricas é o Índice de Percepção de Corrupção (Corruption Perceptions Index - CPI) como proxy para o nível de corrupção. O CPI é divulgado pela Transparência Internacional

\footnotetext{
${ }^{1}$ Tradução livre dos autores: "o uso indevido de cargos públicos para obter ganhos financeiros privados por um funcionário eleito"
} (Kunicová \& Rose-Ackerman, 2005, p.577). 
(Transparency International) que é uma organização não-governamental, com sede situada em Berlim. O índice é constituído pela combinação de 13 bases de dados e classifica o nível de corrupção de um país conforme é percebido por especialistas e executivos empresariais. Ele captura as percepções gerais e pode variar de 0 a 10, quanto maior o valor mais elevado é o nível de corrupção, constituindo um ranking de corrupção entre os países (Transparência Internacional, 2019).

Outro indicador semelhante caracterizado como índices de percepção é International Country Risk Guide (ICRG) produzido pelo grupo PRS, que fornece avaliações e previsões de risco político numa amostra de 140 países (dentre eles desenvolvidos, emergentes e de fronteira). Este índice varia de 0 a 6 de forma que, quanto menor o valor maior a corrupção. $\mathrm{O}$ grupo PRS avalia a corrupção, desde 1980 como um componente do risco político. O índice de corrupção é um dos 22 indicadores de risco de um país produzidos pelo grupo. O ICRG fornece à TI pontuações de corrupção que cooperam na construção do CPI.

Índice de Controle da Corrupção do Banco Mundial (Control of Corruption Index - CCI), é um índice fornecido pelo Banco mundial anualmente. Esse índice é construído a partir de 25 fontes de dados de 20 instituições espalhadas ao redor do mundo, o CCI é um componente dos Indicadores de Governança Mundial (WGI). O CCI pode variar $-2,5$ a $+2,5$ sendo quanto maior a pontuação menor corrupção.

Existem pesquisadores que optaram por construir o próprio índice de corrupção, por exemplo, Dell’Anno e Teobaldelli (2013) optaram por um índice alternativo construído a partir da padronização dos indicadores mais utilizados na literatura: Corruption Perceptions Index (CPI), Worldwide Governance Indicators (WGI), International Country Risk Guide (ICRG), World Development Indicators based on Enterprise Surveys e Enterprise Surveys from World Bank.

No Quadro 3 apresenta-se resumidamente, as principais medidas encontradas nessa pesquisa para a mensuração empírica da corrupção. Na coluna da direita estão elencados os trabalhos que fizeram uso dos respectivos índices em seus estudos.

Quadro 3 - Principais medidas de corrupção identificadas na produção científicas analisadas.

\begin{tabular}{|c|c|c|}
\hline Medida & Fonte de Dados & Relação de artigos que aplicaram o índice \\
\hline $\begin{array}{l}\text { Índice de Percepção de Corrupção } \\
\text { (Corruption Perceptions Index - } \\
\text { CPI) }\end{array}$ & $\begin{array}{l}\text { Elaborado pela Transparência Internacional (TI) } \\
\text { utiliza } 13 \text { fontes de dados de } 11 \text { instituições ao } \\
\text { redor do mundo. } 180 \text { países são avaliados, com } 3 \\
\text { fontes mínimas por país }\end{array}$ & $\begin{array}{l}\mathrm{A} 1, \mathrm{~A} 3, \mathrm{~A} 4, \mathrm{~A} 6, \mathrm{~A} 7, \mathrm{~A} 8, \mathrm{~A} 10, \mathrm{~A} 11, \mathrm{~A} 12, \mathrm{~A} 13, \\
\mathrm{~A} 14, \mathrm{~A} 15, \mathrm{~A} 16, \mathrm{~A} 18, \mathrm{~A} 20, \mathrm{~A} 20, \mathrm{~A} 21\end{array}$ \\
\hline $\begin{array}{l}\text { International Country Risk Guide } \\
\text { (ICRG) }\end{array}$ & $\begin{array}{l}140 \text { países compõe a avaliação do índice. É } \\
\text { composto por } 22 \text { indicadores de risco do país, } \\
\text { sendo o Îndice de Corrupção (IC) um dos } \\
\text { componentes. Elaborado pelo grupo PRS }\end{array}$ & $\mathrm{A} 2, \mathrm{~A} 12$, \\
\hline $\begin{array}{l}\text { Índice de Controle da Corrupção do } \\
\text { Banco Mundial (Control of } \\
\text { Corruption Index-CCI), }\end{array}$ & $\begin{array}{l}\text { Construído a partir de } 25 \text { fontes de dados de } 20 \\
\text { instituições ao redor do mundo, um total de } 212 \\
\text { países são avaliados atualmente }\end{array}$ & A1, A3, A4, A8, A16, A19 \\
\hline Índices alternativos & Construído a partir dos dados das pesquisas & A5, A17 \\
\hline
\end{tabular}

Fonte: Dados da pesquisa.

A literatura analisada aponta como um dos maiores desafios da análise empírica da corrupção, a sua medição (Lederman et al., 2005; Serra, 2006; Pellegrini \& Gerlagh, 2011; Kotera et al. 2012). Constata-se a existência de uma discussão teórica a respeito da confiabilidade dos índices caracterizados como índices de percepção, dentre eles o de corrupção. Pois são tipos de medidas, que se fundamentam em avaliações de natureza subjetivas, nas medidas de percepção do fenômeno na sociedade (Lederman et al., 2005; Kunicová \& Rose-Ackerman, 2005) alguns índices são fornecidos no intuito de oferecer avaliações de risco e governança para investidores internacionais. Por isso, recomenda-se cuidado ao utilizar os índices de percepção em trabalhos empíricos para que não ocorra a distorção de informações, devido o reconhecimento de suas limitações ao interpretar os resultados (Lederman et al., 2005; Serra, 2006;). No entanto, apesar da discussão acerca das limitações desse indicador, há também aspectos positivos que são destacados. Em relação a confiabilidade, a comparação de índices com diferentes métodos tem comprovado esse quesito dos índices de percepção, apesar de não significar necessariamente que tais índices medem 
precisamente a corrupção, no entanto ainda são alternativas para a análise empírica (Lederman et al., 2005; Serra, 2006; Pellegrini \& Gerlagh, 2011; Kotera et al., 2012).

\section{Considerações Finais}

O presente estudo buscou averiguar as principais contribuições de estudos empíricos publicados entre 2005 e 2020, a fim de ampliar a compreensão sobre os fatores causais de corrupção, ou seja, as causas desse fenômeno. Analisou-se 20 estudos empíricos que abordavam pelo menos um antecedente causal da corrupção. Observou-se que pesquisas empíricas acerca da corrupção, avançaram consideravelmente nos últimos anos, tal avanço pode ser atribuído ao surgimento de novas fontes de dados, indicadores e formas de mensuração desse fenômeno conforme apresentados no decorrer da análise. Apesar de ainda existirem divergências e discussões a respeito das formas de mensuração, há também na literatura concordância a respeito de suas colaborações. Avanços na questão da mensuração de corrupção, são importantes uma vez que quanto melhor, mais robustos e confiáveis são os resultados obtidos pelas investigações sobre as causas e efeitos da corrupção, melhor podem ser direcionadas as políticas e ações de enfrentamento e prevenção do fenômeno (Billger \& Goel, 2009, Serra, 2006; Lambsdorff, 2006).

Notou-se a multidisciplinaridade do tema, que assim como o fenômeno da corrupção, a análise do mesmo se apresenta em diversas formas e vertentes. Identificamos três principais perspectivas ou dimensões de estudos, aqueles com princípios e pressupostos ligados a economia, e que cabe ressaltar que tal dimensão pode ser encontrada na literatura acerca das consequências da prática corrupta. Uma segunda perspectiva é a política e legal ajuda na compreensão de aspectos relevantes da corrupção. Por fim, a perspectiva demonstrada é a que abarca questões sociais e culturais como um fator causal da corrupção, e apesar de ainda serem menos numerosos os estudos que analisam a corrupção sobre esse foco, tem crescido nos últimos tempos.

Ao examinar a literatura sobre corrupção observou-se a dificuldade de mensuração ou medição do fenômeno, visto que se trata de atos ilegais ou condenáveis e está acompanhado na maior parte das vezes pelo sigilo e ocultação (Pellegrini \& Gerlagh, 2008). As principais medidas ou indicadores utilizados pelos estudos componentes da base dessa pesquisa são o Índice de Percepção de Corrupção (Corruption Perceptions Index - CPI), o International Country Risk Guide (ICRG) e o Índice de Controle da Corrupção do Banco Mundial (Control of Corruption Index - CCI). Tais índices são usados em 90\% dos estudos analisados. Porém, há alguns estudos que optam pela construção de um índice próprio, aqui também denominado como indicador alternativo, no entanto ainda representam uma pequena parcela dos trabalhos analisados nessa pesquisa.

A partir do entendimento da existência de diversas concepções acerca da definição do fenômeno corrupção, bem como dos atos que a envolvem, é possível classificar a corrupção como um fenômeno complexo, com diversos delineamentos. Nele, as motivações do comportamento corrupto possuem diversos delineamentos e resultam das interações com o ambiente ao qual o ator pertence. Tal complexidade do tema propicia a coexistência de diversas teorias e abordagens que relacionam o estudo das causas e efeitos da corrupção conforme demonstrado anteriormente, assim como os mecanismos de combate, de diferentes maneiras (Brei, 1996).

Como contribuição prática dessa pesquisa ressalta-se a colaboração para o entendimento do estado da arte sobre os fatores causais da corrupção, como já citado anteriormente um fenômeno de características complexas. Nesse sentido, pode-se destacar que há uma evolução e amadurecimento na compreensão do fenômeno da corrupção, e que tal situação pode ser melhor esclarecido por inúmeros fatores políticos, sociais, culturais, econômicos e legais de uma sociedade.

Entre os limites dessa pesquisa se encontra o fato de ter se realizado análise em estudos que buscaram identificar causas da corrupção a um nível macro, e, especialmente, entre países. Portanto, os resultados desse estudo devem ser explorados respeitando o contexto analisado. 
Para pesquisas futuras, destacam-se as possibilidades de realização do estudo das causas de corrupção em níveis micro e intranacionais, analisando as formas de mensuração identificando questões específicas e regionais dos antecedentes causais de corrupção. Ainda, para continuidade dessa pesquisa destaca-se a possibilidade de segui-la de forma empírica verificando in loco variáveis contextuais que podem impactar na incidência de corrupção, e na construção de um constructo local sobre a corrupção.

\section{Agradecimentos}

Agradecemos à Coordenação de Aperfeiçoamento de Pessoal de Nível Superior (CAPES) pelo apoio e incentivo a pesquisa.

\section{Referências}

Abu, N., Karim, M. Z. A. \& Aziz, M. I. A. (2015) 'Corruption, Political Instability and Economic Development in the Economic Community of West African States (ECOWAS): Is There a Causal Relationship? Contemporary Economics, 9(1).

Arapović, A., A. Depken, I. I. \& Hadžikadić, M. (2017) 'Corruption in Transition Economies: Cause or Effect? Zagreb International Review of Economics \& Business, 20(1), 113-123. 10.1515/zireb-2017-0011.

Bardin, L. (2004), Análise de conteúdo. Edições 70.

Avritzer, L. \& Filgueira, F. (2011) Corrupção e controles democráticos no Brasil, Textos para Discussão - CEPAL/IPEA. Naciones Unidas Comisión Económica para América Latina y el Caribe (CEPAL)

Becker, G. S. (1968) ‘Crime and Punishment: An Economic Approach’, Journal of Political Economy, 76(2), pp. 169-217. 10.1086/259394.

Bento, A. (2012). Como fazer uma revisão da literatura: Considerações teóricas e práticas. Revista JA (Associação Académica da Universidade da Madeira), 7(65), 42-44

Billger, S. M. \& Goel, R. K. (2009) 'Do existing corruption levels matter in controlling corruption? Cross-country quantile regression estimates', Journal of Development Economics, 90(2), 299-305. 10.1016/j.jdeveco.2008.07.006.

Brei, Z. A. (1996) 'A corrupção: causas, consequências e soluções para o problema’, Revista de Administração Pública, 30 (3), 103 a 115.

Brei, Z. A. (1996b) “Corrupção: dificuldades para definição e para um consenso”, Revista de Administração Pública. $30(1), 64$ a 77.

Campante, F. R., Chor, D. \& Do, Q.-A. (2009) 'Instability and the Incentives for Corruption', Economics \& Politics, 21(1), 42-92. https://doi.org/10.1111/j.14680343.2008.00335.x.

Dell'Anno, R. \& Teobaldelli, D. (2015) 'Keeping both corruption and the shadow economy in check: the role of decentralization', International Tax and Public Finance, 22(1), 1-40. 10.1007/s10797-013-9298-4.

Fisman, R. \& Gatti, R. (2002) 'Decentralization and corruption: evidence across countries’, Journal of Public Economics, 83(3), pp. 325-345. 10.1016/S00472727(00)00158-4.

Galvão, C. M., Sawada, N. O. \& Trevizan, M. A. (2004) 'Systematic review: a resource that allows for the incorporation of evidence into nursing practice', Revista Latino-Americana de Enfermagem, 12(3), 549-556. 10.1590/S0104-11692004000300014.

Goel, R. K. \& Nelson, M. A. (2010) 'Causes of corruption: History, geography and government', Journal of Policy Modeling, 32(4), 433-447. 10.1016/j.jpolmod.2010.05.004.

Judge, W. Q., McNatt, D. B. \& Xu, W. (2011) 'The antecedents and effects of national corruption: A meta-analysis', Journal of World Business, 46(1), 93-103. 10.1016/j.jwb.2010.05.021.

Kotera, G., Okada, K. \& Samreth, S. (2012) 'Government size, democracy, and corruption: An empirical investigation', Economic Modelling, 29(6), 2340-2348. 10.1016/j.econmod.2012.06.022.

Kunicová, J. \& Rose-Ackerman, S. (2005) 'Electoral Rules and Constitutional Structures as Constraints on Corruption', British Journal of Political Science, 35(4), 573-606. 10.1017/S0007123405000311.

Lambsdorff, J. G. (2006) Causes and Consequences of Corruption: What Do We Know from a Cross-Section of Countries? International Handbook on the Economics of Corruption. Edward Elgar Publishing.

La Porta, R. et al. (1999) 'The Quality of Government', Journal of Law, Economics, \& Organization, 15(1), 222-279.

Laville, C \& Dionne, J. (1999), A construção social do saber: manual de metodologia da pesquisa em ciências humanas. Editora UFMG.

Lederman, D., Loayza, N. V. \& Soares, R. R. (2005) 'Accountability and Corruption: Political Institutions Matter', Economics \& Politics, 17(1), 1-35. https://doi.org/10.1111/j.1468-0343.2005.00145.x 
Nascimento, L. N. do (2018) 'Mais capacidade estatal, menos corrupção? Uma análise para a América Latina (1996-2015)', Revista de Sociologia e Política, 26(68), pp. 95-120. 10.1590/1678987318266806.

Nascimento, J. C. H. B. do, Macedo, M. A. da S., Siqueira, J. R. M. de \& Neto, A. R. (2019) “Corrupção governamental e difusão do acesso à Internet: evidências globais", Revista de Administração Pública. 53(6), 1011-1039.

North, C. M., Orman, W. H. \& Gwin, C. R. (2013) 'Religion, Corruption, and the Rule of Law', Journal of Money, Credit and Banking, 45(5), 757-779. https://doi.org/10.1111/jmcb.12024.

Pellegrini, L. \& Gerlagh, R. (2008) 'Causes of corruption: a survey of cross-country analyses and extended results', Economics of Governance, 9(3), 245-263. $10.1007 / \mathrm{s} 10101-007-0033-4$.

Policardo, L. \& Carrera, E. J. S. (2018) 'Corruption causes inequality, or is it the other way around? An empirical investigation for a panel of countries', Economic Analysis and Policy, 59(C), 92-102. 10.1016/j.eap.2018.05.001.

Policardo, L., Sanchez Carrera, E. J. \& Risso, W. A. (2019) 'Causality between income inequality and corruption in OECD countries', World Development Perspectives, 14, p. 100102. 10.1016/j.wdp.2019.02.013.

Palifka, B. J. \& Rose-Ackerman, S. (eds) (2016) 'What Is Corruption and Why Does It Matter?', in Corruption and Government: Causes, Consequences, and Reform. 2nd edn. Cambridge: Cambridge University Press, 3-48. 10.1017/CBO9781139962933.003.

Reis, A. de O., Almeida, F. M. de \& Ferreira, M. A. M. (2018) "Relações entre Corrupção Percebida e Transparência Orçamentária: um estudo com abordagem cross-national", Revista Contemporânea de Contabilidade, 15(37), 158-177. 10.5007/2175-8069.2018v15n37p158.

Serra, D. (2006) 'Empirical determinants of corruption: A sensitivity analysis', Public Choice, 126(1), 225-256. 10.1007/s11127-006-0286-4.

Tang, Z. et al. (2019) 'The effects of social media use on control of corruption and moderating role of cultural tightness-looseness', Government Information Quarterly, 36(4), 101384. 10.1016/j.giq.2019.06.001.

Tanzi, V. (1998) 'Corruption Around the World: Causes, Consequences, Scope, and Cures', Staff Papers (International Monetary Fund), 45(4), 559-594. $10.2307 / 3867585$.

Themudo, N. S. (2014) 'Government Size, Nonprofit Sector Strength, and Corruption: A Cross-National Examination', The American Review of Public Administration, 44(3), 309-323. 10.1177/0275074012465791.

Treisman, D. (2000) 'The causes of corruption: a cross-national study', Journal of Public Economics, 76(3), 399-457. 10.1016/S0047-2727(99)00092-4.

Van Rijckeghem, C. \& Weder, B. (2001) 'Bureaucratic corruption and the rate of temptation: do wages in the civil service affect corruption, and by how much?', Journal of Development Economics, 65(2), 307-331. 10.1016/S0304-3878(01)00139-0.

Viana, C. J. P. et al. (2020) 'Efecto conjunto de la democracia y libertad económica sobre la corrupción', Revista de Administração Pública, 54(2), 285-300. 10.1590/0034-761220190165.

Vergara, S.C. (2006), Projetos e relatórios de pesquisa. Atlas. 